\title{
IMPROVING THE SYNCHRONIZATION OF OFDM SIGNALS IN THE SYSTEM DVB-T2
}

\author{
S. M. Al-Merahi, \\ Sana's University, Sana, Yemen
}

DOI: 10.36724/2664-066X-2020-6-2-2-6

\begin{abstract}
Describe the principles of synchronization in systems of digital television broadcasting DVB-T2 and TDS-OFDM (DTTB), which provide all forms of synchronization with the signal analysis in time and frequency domains. In the system TDS-OFDM guard interval to obtain the pseudorandom sequence - m-sequence, which is used for synchronization and estimation of channel state. It allows to provide high interference immunity of the system and the acceleration of the synchronization process. It is proposed to use m-sequence in the system of DVB-T2 with placing it in the protective interval. The results of the modeling process correlation of $\mathrm{m}$-sequence, transmitted together with the information of the cyclic prefix.
\end{abstract}

KEYWORDS: sinchronization, OFDM signals, DVB-T2, TDS-OFDM, DTTB.

\section{INTRODUCTION}

In the process of receiving OFDM signals, accurate synchronization of the receiver and transmitter in time and frequency is important. High-quality synchronization can significantly increase the receiver's noise immunity. The DVBT2 synchronization system provides all forms of synchronization with signal analysis in the time and frequency domain - OFDM frames, symbols, time position of sampling intervals and frequency synchronization of subcarriers.

Synchronization in the time domain requires large hardware costs and allows one to achieve a lower accuracy of estimates, therefore it is used at the stage of rough estimation of time and frequency mismatch, and for an accurate estimate, algorithms in the frequency domain are used to a large extent.

Synchronization at the receiver is performed prior to demodulating the symbol subcarriers. The receiver must obtain information about the exact time position of the symbol to minimize the effects of intersymbol interference (ISI) and inter-subcarrier interference (ICI).

The proposed method of using PSP (m-sequences) in the DVB-T2 system will retain the possibility of using QAM-256 modulation while improving the quality of synchronization and its acceleration. 


\section{Synchronization principles in DVB-T2 system}

For synchronization in the DVB-T2 system, P1 and P2 symbols are used (Fig. 1), as well as pilot signals located in the OFDM frame. P1 marks the start of a frame, and P2 follows P1 in every frame and provides access to physical layer PLPs in every frame. Several transport streams (TV, radio, HDTV, SD, mobile communication) can be transmitted in the DVB-T2 channel simultaneously, each of them being placed in its own PLP channel.

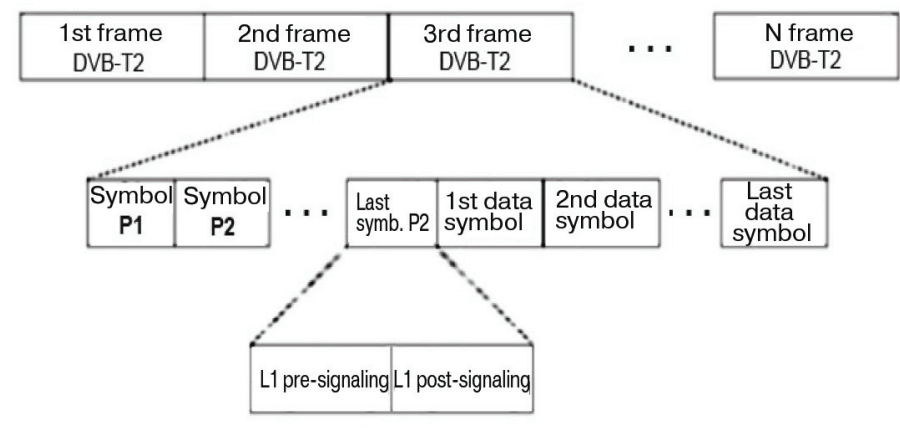

Figure 1. Placing P1, P2 symbols and L1 signals in a DVB-T2 frame [1]

Sampling clock synchronization is also performed, which is based on the information contained in the P1 symbol.

P1 symbol is used for synchronization and identification of DVB-T2 stream. It also contains information about the DVB-T2 frame - the number of subcarriers in OFDM (1k-32k) and the transmission format of the P2 symbol following P1 (MISO or SISO modes). Element P1 is an OFDM symbol with DBPSK modulation, two guard intervals on both sides (in the sum of $1 / 2$ symbol duration).

Frequency synchronization is necessary to protect symbols from inter-subcarrier interference (ICI) and to maintain orthogonality in OFDM symbols. Orthogonality is violated when the carrier frequency shift appears, which appears in the absence of synchronization of the frequency generators on the transmitting and receiving sides. In addition, the frequency shift occurs due to the Doppler effect [1].

Sampling clocks are equally important. The offset of the sampling interval leads to severe distortion of the reconstructed OFDM signal. These distortions occur in multipath reception with fading, noise, and timing errors in symbol position. The sampling interval offset is divided into a sampling phase offset and a sampling frequency offset [2].

Let's consider the sequence of synchronization in the DVB$\mathrm{T} 2$ receiver. The synchronization process begins with the detection of the P1 symbol. After the P1 symbol is detected, information about the symbol timing and frequency and the size of the fast Fourier transform (FFT) OFDM is obtained from it. After determining the size of the Fourier transform, the presence of the guard interval (GI) is determined. To detect the pilot signals, the basic information signal (OFDM symbols) is used and the L1 signal in P2 symbols is decoded. Since the receiver already knows the FFT length at this time, the receiver detects the pilot signals and can decode the data located at P2 and L1.
By decoding the signaling information in L1, the receiver determines the layout of the pilots and GIs in the OFDM data symbol stream. P1 marks the beginning of each frame. Detection of the P1 symbol is sufficient to start processing the DVB-T2 RF carriers. In addition, the P1 symbol is used to correct time and frequency synchronization.

The received radio signal, transmitted from the transmitting to the receiving antenna, may have a certain frequency shift $\Delta \mathrm{f}$, when the transmitter and receiver move relative to each other the Doppler effect. The frequency shift of the OFDM signal results in a loss of orthogonality between the subcarriers of the OFDM symbols, which causes a decrease in the quality of the demodulation of the received signal.

Receiving signals in urban environments with multi-beam signal propagation and Rayleigh fading is especially difficult in solving synchronization problems. Such signal propagation leads to significant distortion of its spectrum and complicates the task of synchronization and estimation of channel parameters in the frequency domain.

The task of frequency synchronization is to estimate and correct the frequency shift $\Delta \mathrm{f}$ with the required accuracy. Since a pair of Fourier transforms is used to generate and allocate orthogonal subcarriers in OFDM systems, signals are generated and transmitted as OFDM symbols. To minimize the effects of intersymbol interference in multipath reception, guard intervals are introduced between individual symbols.

Eliminating the loss of orthogonality of subcarriers during demodulation requires accurate phase and frequency matching of the transmitter and receiver over the entire frequency band of received signals. Phase and frequency mismatch is due to some spread and instability in the frequencies of the transmitter and receiver reference oscillators and Doppler shift in mobile communications.

The effect of synchronization errors increases with the number of subcarriers. Therefore, in order to minimize the phase distortion of the signal on the receiving side, and, consequently, the probability of error during decoding in DVD-T2, high requirements are imposed on time and frequency synchronization.

The use of such complex synchronization with pilot processing requires a significant amount of decision time and comprehensive synchronization of the DVD-T2 receiver. Synchronization time reduction was achieved in the TDS-OFDM system.

\section{Description of the system receiving part TDS-OFDM (DTTB)}

In China, a terrestrial digital TV and multimedia broadcasting (DTMB) system has been developed, which has shown good results and is accepted for use in some countries. In this system, no cyclic prefix is transmitted in the guard interval. It is replaced by a pseudo-random sequence (PRS) that is used for synchronization.

In the TDS-OFDM receiver, the radio frequency signal is fed to the tuner (Fig. 2), converted into an intermediate frequency signal, fed to the ADC and passed through the synchronization blocks (18), the channel equalizer (20), demodulation (22), the precorrection decoder (24). The basic configuration of the receiver includes the following types of subcarrier modulation: QPSK, 16QAM, 64QAM. 


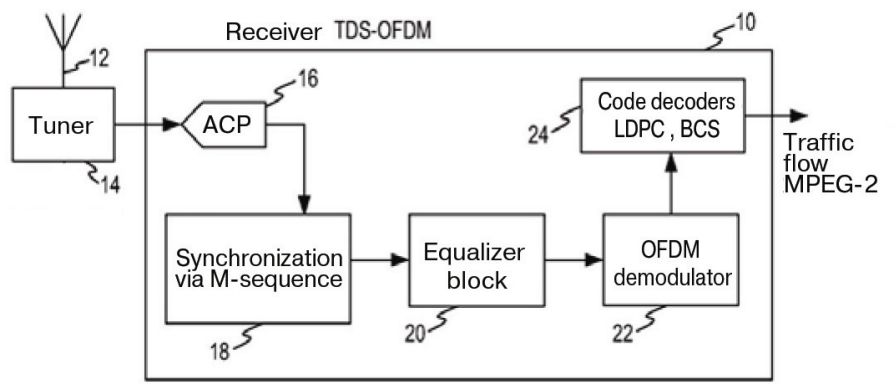

Figure 2. Generalized scheme of the TDS-OFDM receiver with blocks of synchronization, equalizer, OFDM demodulator and an LDPC decoder. The output of the receiver generates transport stream MPEG-2 or MPEG-4

In the TDS-OFDM system, a pseudo-random sequence (PRS), an m-sequence, which is used for synchronization and channel estimation, is shuffled into the guard interval.

Time synchronization of TDS-OFDM symbols is used to isolate the starting point of each symbol, which is necessary for accurate signal sampling. Symbol time offset (STO) estimation is performed in either the time domain or the frequency domain.

To synchronize symbols in the receiver, a training $\mathrm{m}$ sequence is used, which allows you to quickly restore the frame and temporal relationships in the received digital signal, and to make a more reliable estimate of the channel state. Since no pilot signals are transmitted in TDS-OFDM (DTTB), all subcarriers are informational. This increases the system information capacity.

The DTTB TDS-OFDM modulation scheme contains 3780 symbols representing the result of the fast Fourier transform of size 3780 . To ensure high noise immunity, the system uses precorrection with LDPC and BCS codes. Three LDPC codes are available at different rates.

Time estimation (timing) is performed by the correlation signal (block 116). The frequency response of the channel is obtained by taking the FFT from the impulse response of the channel (Fig. 3).

After correlator block 116 and channel estimator 118, OFDM symbols are detected and recovered. During modulation (at the transmitter), the PRS is placed in the guard interval, replacing the usual cyclic prefix. At the receiver, block 120 removes the PRP from the guard interval and recovers the OFDM symbol sequence. The reconstructed OFDM symbols are further processed in the frequency domain by a one-tap equalizer (block 124) with the elimination of the frequency response channel irregularities resulting from fading and multipath reception.

In the multi-subcarrier mode, the reconstructed symbols are time domain data and are then fed to an FFT (122) where a Fourier transform converts these symbols into corresponding frequency domain signals. Block 122 performs a 3780-point FFT.

Before synchronization starts, block 106 receives and processes the IF signal and generates a baseband signal. Then the signals are processed by the PRS correlator (116), which outputs a cross-correlation signal by comparing the incoming PRP and the same sequence generated by the local generator.

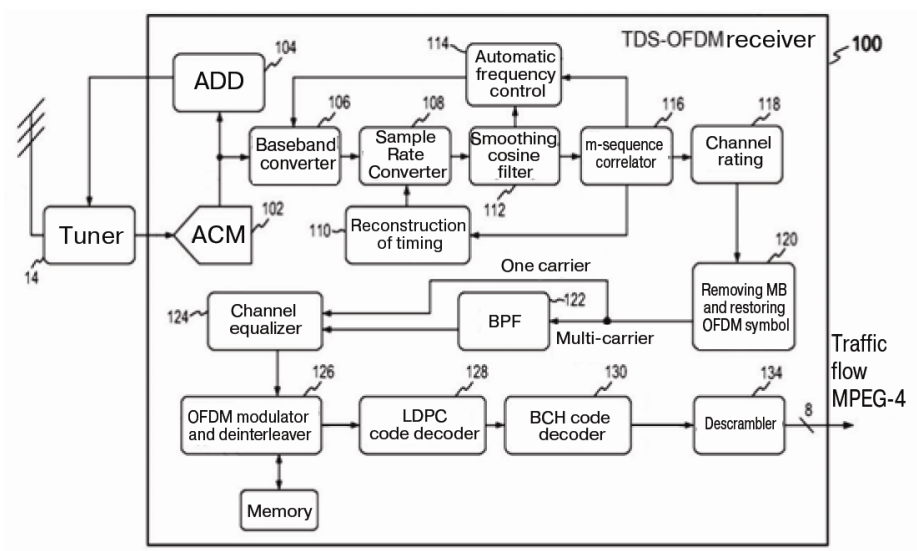

Figure 3. TDS-OFDM receiver circuit

The correlator signal (block 116) is used to reconstruct the carrier, digital signal timing, and parameters used to estimate the channel state. The peak of the function generated by the correlator (Fig. 6,7) marks the beginning of each frame of the OFDM signal, the frequency shift under the Doppler effect, and corrects the temporal relations.

In equalizer 124, a frequency domain response (AFC) is obtained by taking an FFT of a time domain response (channel impulse response). The equalizer estimates the parameters and equalizes the spectral components of the signal in the frequency domain of the channel.

The OFDM demodulator unit (126) reconstructs the OFDM symbols in the signal frame and converts the OFDM symbols to the frequency domain. The error precorrection decoder units (128 and 130) process the LDPC and BCH codes and generate a signal with erroneously received bits corrected.

The M-sequence located in the GI provides faster synchronization than in DVB-T2, fast frame and timing recovery, and reliable channel estimation. This sequence is transmitted to DTTB using spread spectrum technology and is used as a training sequence that increases the efficiency of channel condition estimation and for its correction on reception and accelerates receiver synchronization.

Figure 4 shows the data of a TDS-OFDM frame with a bandwidth of $420(255,165)$, expressed in the number of chips. In addition, variants of the PRP with the length of 511 (84) and 511 (434) are possible with the same frame length 3744 (information symbols) + 36 (TSP symbols). Thus, the PRS and postambulum are placed on the guard interval. In general, a preamble and a postamble can be placed on the guard interval. The PRS themselves (m-sequences) have a length of 255, 511, and the second numbers $(165,84$, and 434$)$ represent the auxiliary parts of these noise sequences.

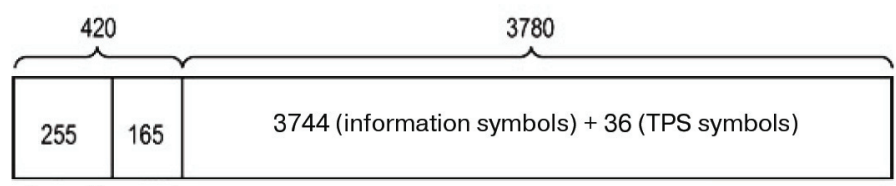

Guard interval

OFDM cascade symbols

Figure 4. Schemes of the OFDM frame with the TDS-OFDM SRP located in the guard interval 
The 3780 frame symbols consist of 3744 basic information symbols and 36 symbols for transmission parameter signals (TPS), which provide information to the receiver demodulator about the rate and length of the interleaving code.

Despite the high performance, the TDS-OFDM (DTTB) system does not allow the transmission of information with QAM256 modulation, sensitive to interference, due to the appearance of mutual interference between the PSP and information symbols [3]. To reduce the appearance of interference in the TDS-OFDM (DTTB) system, in particular, the use of not one, but two PSS located in the GI is provided. Research is underway to completely eliminate the interference between the PRS and symbols.

\section{Using guard interval to improve timing accuracy in DVB-T2}

Synchronization improvements in DVB-T2 can be obtained by placing a pseudo-random sequence in the guard interval (possibly together with a cyclic prefix), as is done in the DTMB system. Unlike the DTMB system, the DVB-T2 system retains all pilot signals during the OFDM symbol and retains the initial and final portions of the cyclic prefix during the RFI. The rest of the prefix is required to terminate the transient at the beginning and at the end of each information character. The pseudo-random sequence is mixed in with the CPU or inserted in place of the cyclic prefix. The use of the PSP provides faster and more accurate symbol synchronization.

Since the weak point of the DTMB system is the interference between the PSP and a block of OFDM symbols [4], it is possible to apply the method of eliminating it proposed in $[5,6]$. It

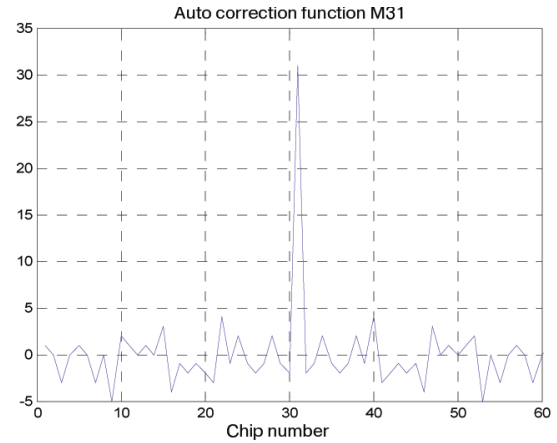

consists in the use of additional pilot signals transmitted as part of information signals, and the method of "compressed sensor" (compressive sensing) for channel estimation in the timefrequency domain (Fig. 5). After the introduction of this method, the DTMB system can use QAM-256 modulation.

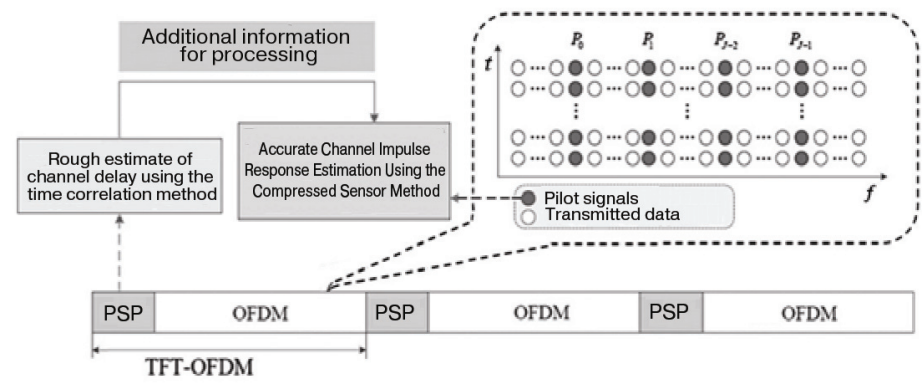

Figure 5. DTMB OFDM frame structure for channel estimation in time-frequency domains and for eliminating interference using PRP

Since in the proposed DVB-T2 structure, after mixing the PRS into the guard interval, the pilot signals are not excluded from the OFDM frame, no additional pilot signals are required. In DVB-T2, after the introduction of PRS and the abovementioned method of eliminating interference, a high-quality QAM-256 transmission is possible. Shorter m-sequences (31 or 63 in length) can be used to reduce crosstalk between the PRS and the OFDM symbol block. They have a fairly high level of the correlation function burst relative to the side lobes (Fig. 6).

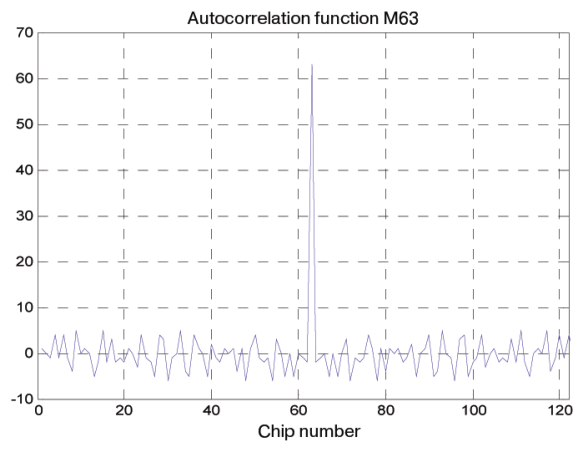

b

Figure 6. Autocorrelation functions for m-sequences of length 31 chips (a) and 63 chips (b) obtained by simulation in Matlab environment

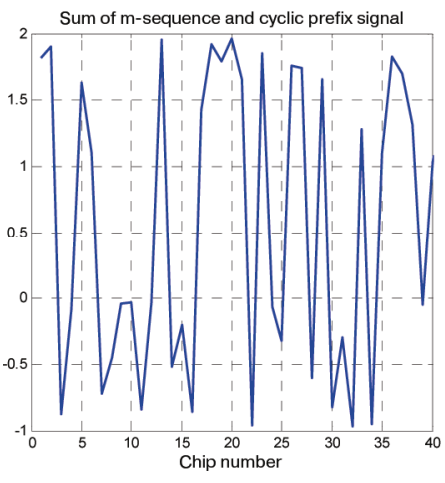

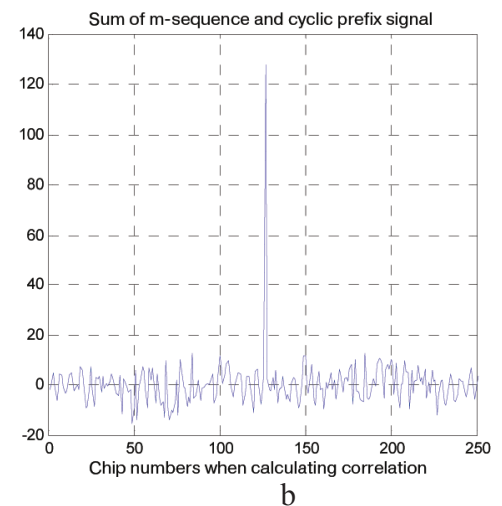

b

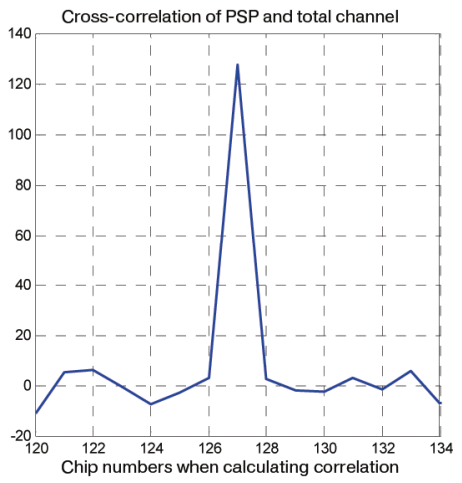

$\mathrm{c}$

Figure 7. Signal after mixing the m-sequence (PSP with a length of 127 chips) to the CPU in the guard interval of the OFDM symbol (a) and signals at the correlator output $(\mathrm{b}, \mathrm{c})$ with different scales along the $\mathrm{x}$ axis 
These PRS can be placed not in the center of the guard interval, but in its end. This will provide better protection against mutual interference between the PRS and OFDM symbol at high multipath delays.

The duration of the sequence is chosen slightly less than the duration of the guard interval in order to ensure attenuation of transients at the end of the first symbol and at the beginning of the next (Fig. 9).

It is possible to eliminate the effect of mutual interference between the PRS and the informational part of the OFDM symbol in the place of their joining by choosing the sequence duration somewhat less than the GI duration in order to ensure the decay of the transient processes at the end of the first symbol and at the beginning of the next one (Fig. 6). Offsets of the PRS to the end of the guard interval will help protect symbols for long delays in multipath environments.

Figure 7 shows the oscillograms after mixing the m-sequence (127 chips long) into the guard interval of the OFDM symbol while maintaining the cyclic prefix signal with subsequent processing of the total signal by the correlator on the receiving side. Modeling was performed in Matlab environment.

An m-sequence 127 chips long was used as a pseudo-random sequence, and another random sequence was taken as a CPU. It is obvious that the CPU signal, which is chosen as complex, does not affect the quality of the formation of the peak of the correlation function, which significantly exceeds the level of the side lobes. In order to reduce the level of the total signal, the amplitude of the m-sequence can be reduced by up to five times. In this case, the correlation function has a sufficient amplitude.

The cyclic prefix (CPU - signal copied into the guard interval) improves the transient at the beginning of each OFDM symbol. However, from this point of view, there is no need to copy the entire CPU, since the memory bandwidth and the information part of the symbol are spaced in time. Transients in a channel with a bandwidth of more than $7 \mathrm{MHz}$ decay with a duration of less than $1 \mu \mathrm{s}$. Therefore, you can not transfer the entire CPU, but insert the start and end of the CPU at the end of the first character and at the beginning of the next character, respectively.

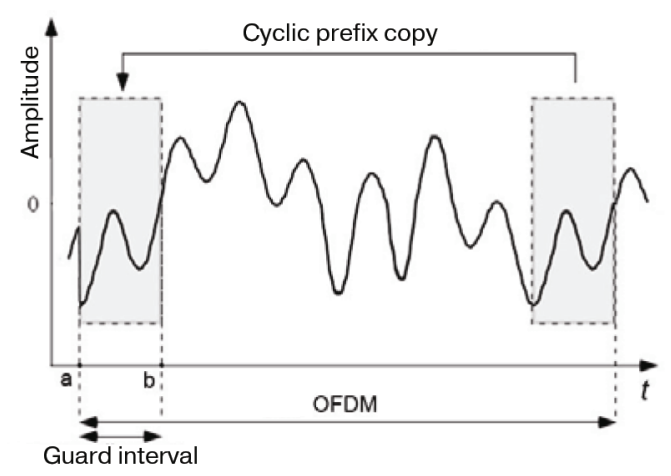

Figure 8. OFDM symbol with a guard interval and with CPU copying from the end of the symbol to this guard interval

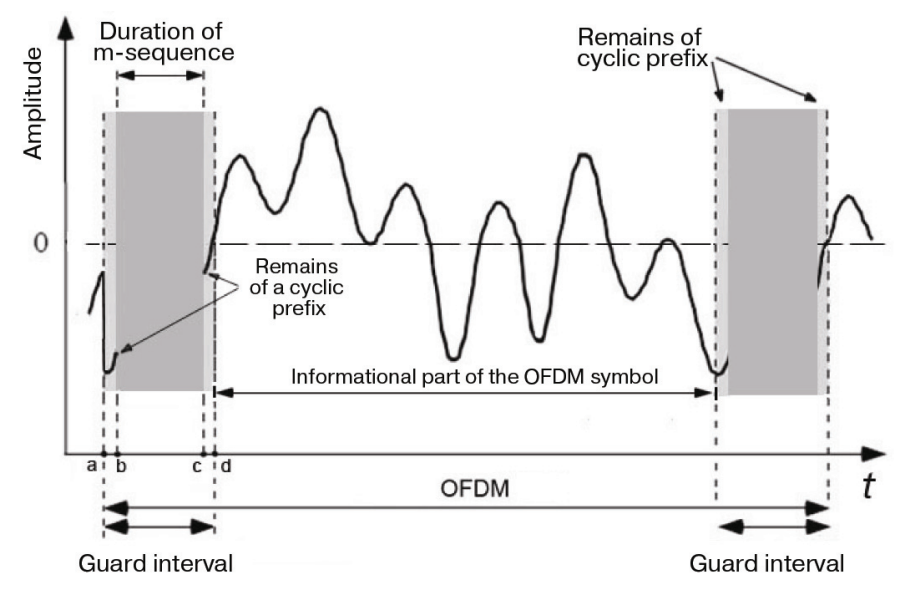

Figure 9. OFDM symbol with guard interval and partial CPU copying and freeing up space for pseudo-random sequence placement

Figures 8 and 9 show one OFDM symbol with a guard interval and with CPU copying from the end of the symbol to this guard interval. Figure 9 shows an OFDM symbol with a guard interval and partial copying of the CPU and freeing up space for placing an additional signal.

\section{Conclusion}

The proposed method of using PSP (m-sequences) in the DVB-T2 system will retain the possibility of using QAM-256 modulation while improving the quality of synchronization and its acceleration. Preservation of the main elements of the system without fundamental changes, in particular, preservation of all pilot signals transmitted in the OFDM frame, will ensure the compatibility of the modified DVB-T2 system with the existing one and will allow receiving signals using the bandwidth to old DVB-T2 receivers with a gradual increase release of new receivers with better synchronization.

\section{References}

1. Morshed Md. S. Synchronization Performance in DVB-T2 System. Tampere university of technology. Department of communications engineering, 2009.

2. Yang L., Venkatachalam D. Receiver For An LDPC based TDSOFDM Communication System. Патент № US 20080025424 A1. 2008.

3. Dai L., Wang J., Wang Z. Time Domain Synchronous OFDM Based on Simultaneous Multi-Channel Reconstruction. Tsinghua National Laboratory for Information Science and Technology, Tsinghua University, China, 2013.

4. Bagade A. B., Deshmukh A. Comparison on CP-OFDM and TDS-OFDM Using Compressive Sensing Theory in Wireless Systems. Journal of Innovation in Electronics and Communication Engineering, Vol. 5(2), July - Dec 2015.

5. Yang F., Ding W., Dai L. Joint Time-Frequency Channel Estimation Method for OFDM Systems Based on Compressive Sensing. IEEE. Research Institute of Information Technology. China. 2014.

6. Jain S. Time-Frequency Training OFDM using Matlab for high speed environments. International Journal of Scientific and Research Publications, Vol. 3, Issue 7, July 2013. 